\title{
CRITICAL SUCCESS FACTORS FOR DATA WAREHOUSING: A CLASSIC ANSWER TO A MODERN QUESTION
}

\author{
James F. Kimpel, Robert Morris University,jfkst14@mail.rmu.edu
}

\begin{abstract}
Businesses, operating in an increasingly complex world, are pressured by globalization, customer demand, government regulations, market conditions, and competition. Executives must have the right data to make strategic, tactical, and operational decisions. Data warehouses, when implemented correctly, promise to meet this need by integrating and organizing key operational data in a form that is consistent, reliable, timely, and readily available, wherever and whenever needed. Unfortunately, 40\% to 50\% of data warehouse initiatives end in costly failure. The search for root causes converged on not understanding the user's business problems. Rockart's classic Critical Success Factor (CSF) method offers a proven way to move from high-level need to the lower-level data that support management decisions. While this paper summarizes the research on data warehousing implementation CSFs, its primary focus is to recommend Rockart's CSF method as a means to address the fundamental challenge of building an effective data warehouse; namely understanding the user's business problems and providing concise, dependable, data, information, and knowledge, about current trends and changes. The outcomes may be useful to business leaders and information technologists as they plan and implement data warehousing (DW) solutions.
\end{abstract}

Keywords: Critical Success Factors (CSFs), Data Warehouse (DW), Business Intelligence (BI) and Information System Implementations

\section{INTRODUCTION}

The environment in which firms operate is growing in complexity [3]. Since the 1900's nearly three billion people have become players in the global stage, overcoming cultural, religious, ethnic, and political barriers to market entry [9]. In addition to globalization, other environmental factors that are pressuring businesses to perform at high levels include: customer demand, government regulations, market conditions, and competition [3]. To meet the performance challenge, companies require considerable volumes of timely, relevant, high quality data, information, and knowledge [3]. Executives must have the right data to make strategic, tactical, and operational decisions.

It is prudent at this point to delineate the difference between data, information, and knowledge. Debons [7] provides the following descriptions that shall be used throughout this article, "... the common view holds that data are composed of the simple measurements around us, information is built out of an organization of data, and knowledge is built out of patterns in the information." With preliminary definitions in place, this article proceeds by elaborating on the executive's need for data, information, and knowledge. Next, this paper considers the data warehousing solution to the executive's need. When implemented well, data warehouses provide strategic value. Unfortunately, up to $50 \%$ of data warehousing initiatives fail [18]. The high failure rates prompted a fundamental research question: "What methods exist to help assure data warehouse implementation success?" This paper summarizes the search for root causes of failures, which converged on the primary root cause of not understanding the user's business problems, and recommends Rockart's [16] Critical Success Factor (CSF) method as a proven approach to move from the higher-level problem to lower-level answer.

\section{The Need}

The need to provide decision support data to executives is not new. In 1961, Daniel wrote a Harvard Business Review article titled "Management Information Crisis" [6]. His article began with three vignettes of companies who were struggling to make management decisions because they lacked quality information. He observed,

"In retrospect it is obvious that these three companies were plagued by a common problem: inadequate management information. The data were inadequate, not in the sense of there not being enough, but in terms of relevancy for setting objectives, for shaping alternative strategies, for making decisions, and for measuring results against planned goals [6]. 


\section{Issues in Information Systems \\ Volume 14, Issue 1, pp.376-384, 2013}

Daniels response to the "Management Information Crisis" was a call for a data warehouse.

\section{The Data Warehouse Solution}

Turban, Sharda, Delen, and King [3] agreed with Daniel and noted, "Decision makers require concise, dependable, information about current operations, trends, and changes." Kimball [12] concurred when he stated, "The basic mission of a data warehouse professional ... is to publish the right data." The data, however, is often fragmented by functional silos [14]. Rockart [16] described the problem as, "Once one gets above the functional level, there is a wide variety of information that one might possibly need, and each functional specialty has an interest in 'feeding' particular data to a general manager.... a massive information flow occurs." Figure 1 illustrates the silo effect.

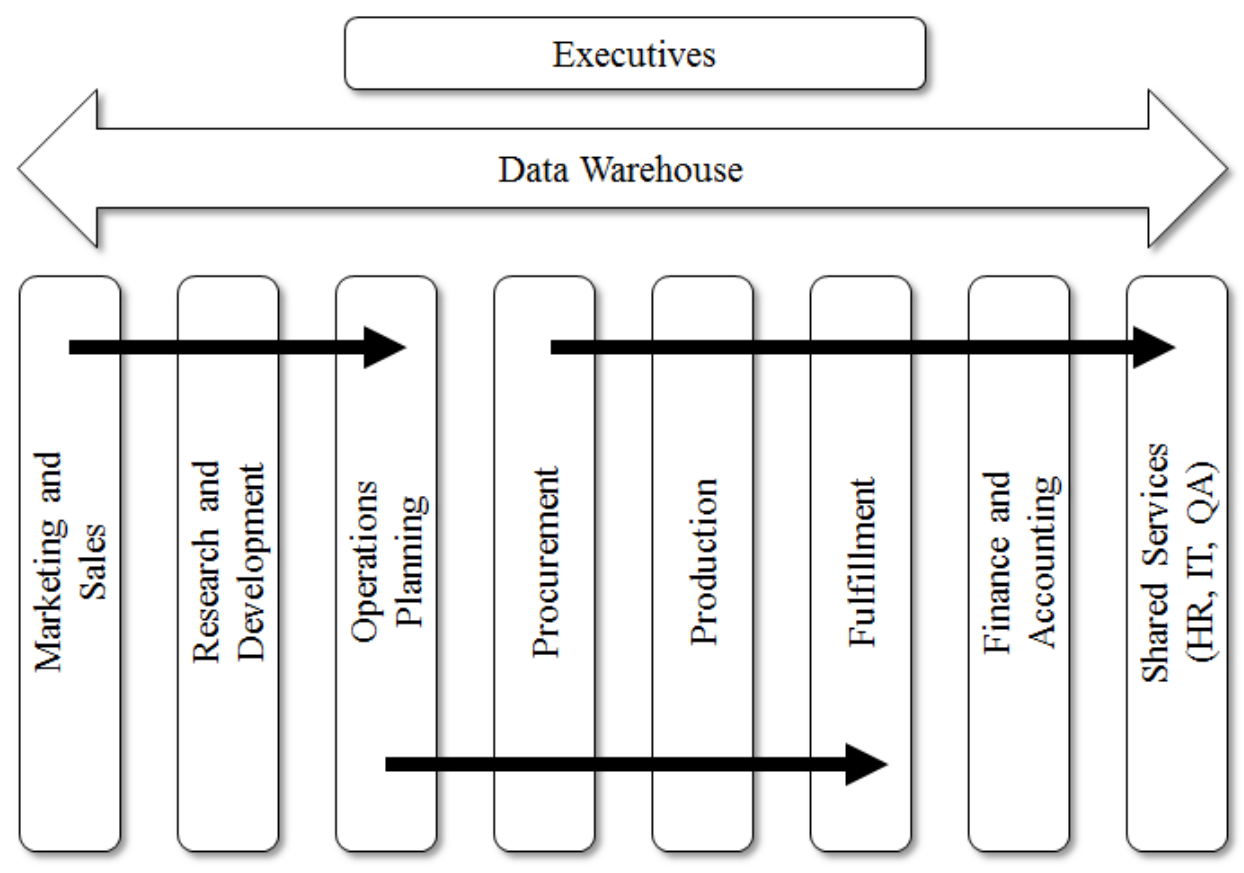

Figure 1. The Silo Effect

Fortunately, "Data warehousing cuts through the obstacle by accessing, integrating, and organizing key operational data in a form that is consistent, reliable, timely, and readily available, wherever and whenever needed" [3]. Connor [4] concurred and noted, "A data warehouse consolidates data and information from multiple corporate wide departments." Turban et al., [3] define a data warehouse as, “... a pool of data produced to support decision making, it is also a repository of current and historical data of potential interest to managers throughout the organization. Inmon [11] stated data warehouses were: subject oriented, integrated, time variant, and non-volatile.

\section{Data Warehouse Success}

Sen, Ramamurthy, and Sinha [18] observed, "Data warehousing has experienced tremendous growth in the last decade [2002 - 2012]. It has become so popular in industry that it was cited as the highest priority postmillennial project of more than half of IT executives." Hwang and Xu [10] agreed when they noted,

Since the early 1990s, the data warehouse has become the foundation of advanced decision-support applications. Using sophisticated business intelligence tools, some corporations are able to use insights gained from their data warehouse to significantly increase sales, reduce costs, and offer new and better products or services.

A Gartner Research survey of CIOs found, “... BI [Business Intelligence] implementation will be a significant factor in delivering IT's contribution to business growth" [8]. A survey of 98 data warehousing professionals conducted 


\section{Issues in Information Systems \\ Volume 14, Issue 1, pp.376-384, 2013}

by Hwang and $\mathrm{Xu}[10]$ using a Likert scale $(1=$ not significant, $2=$ somewhat significant, $3=$ significant, $4=$ very significant, 5 = extremely significant) found the following benefits in successful data warehouse initiatives (Figure 2):

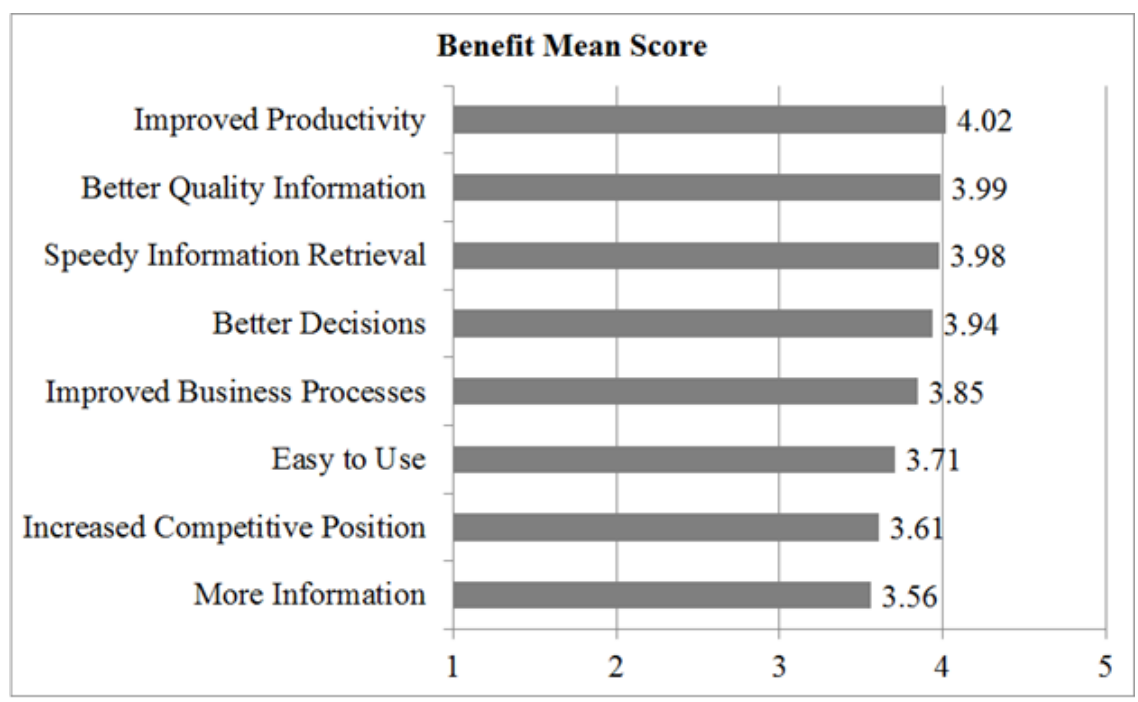

Figure 2. Successful Data Warehouse Benefit Mean Score

\section{Data Warehousing Failure}

Despite the booming data warehousing market, a large number of costly data warehouse initiatives are ending in failure [18]. Connor [4] estimated failure rates at 40\%. Gartner Research [8] and Sen et al. [18] foresaw failure rates as high as $50 \%$. Hwang and $\mathrm{Xu}[10]$ commented, “... stories abound about expensive and failed implementations." Even data warehouse projects that avoid failure often produce suboptimal results with limited strategic benefit to the firm [8]. MacDonald [13] noted, “... building a data warehouse is one of the most complex processes a corporation can undertake." Kimball [12] concurred and stated, "Perhaps more than any other job in IT, the data warehouse design task combines computer technology, cognitive psychology, business content, and politics."

\section{The Search For Causes OF Failures And For CSFs}

In response to data warehouse implementation challenges, several researchers undertook studies aimed not only at uncovering the causes of failures, but also at identifying success factors. This section of the paper highlights the results of several studies. The information is presented from oldest to newest.

MacDonald [13] offered five high level recommendations based on his professional experience:

1. Focus on a real problem.

2. Appoint a data warehouse champion.

3. Use detailed historical data.

4. Apply technology to the business.

5. Trust the data - history does not lie.

Hwang and $\mathrm{Xu}[10]$ conducted a literature review and found $11 \mathrm{CSF}$ for data warehouse projects, and then conducted a survey to determine the relative importance of each CSF. Their survey results $(n=98)$ are presented in Figure 3. The top three CSFs were: source-data quality, clearly defined business needs/benefits, and measureable business benefits [10]. It is interesting to note that two of the top three CSFs were focused on understanding the business context in which the data warehouse would operate. 


\section{Issues in Information Systems}

Volume 14, Issue 1, pp.376-384, 2013

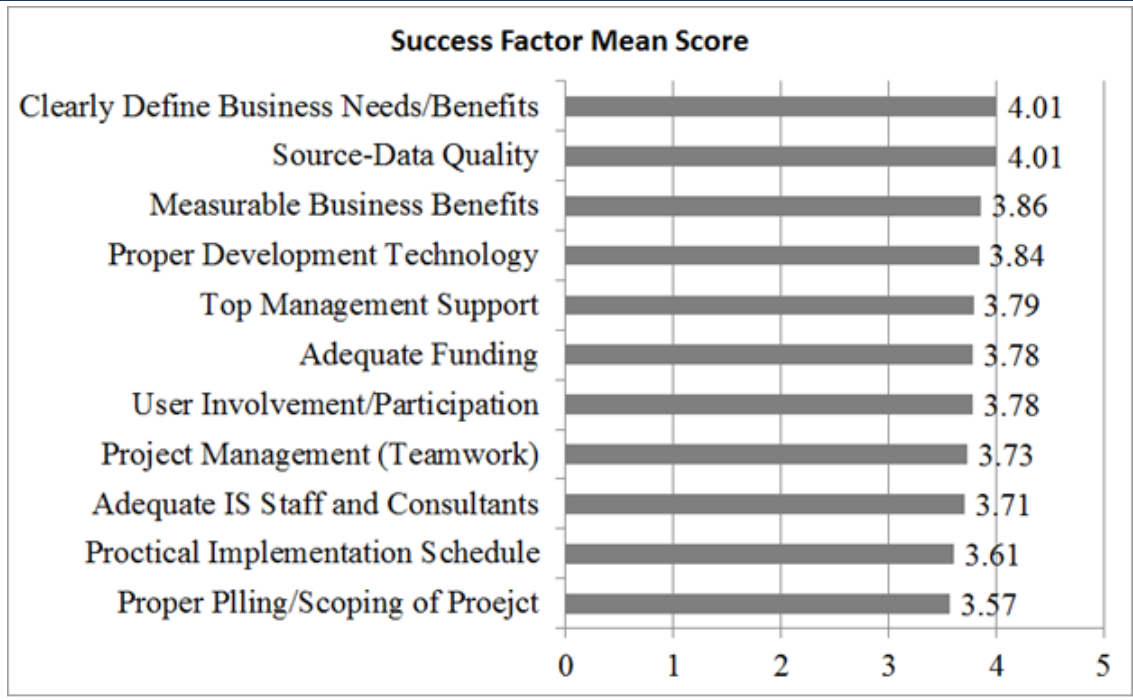

Figure 3. Success Factor Mean Score

BI systems are similar to data warehouses and are typically composed of three complementary components; data warehousing, online analytical processing (OLAP), and data mining tools [20]. BI can be defined as, “... solutions that are responsible for transforming data into information and knowledge, and they also create an environment for effective decision - making, strategic thinking, and acting in organizations" [15].

In 2008, Yeoh, Koronios, and Gao [20] observed empirical research regarding CSFs for BI system implementations was limited. Accordingly, they conducted research (which included a Delphi panel, interviews, and a quantitative survey) into CSFs affecting implementations [20]. A summary of their findings may be found in Figure 4.

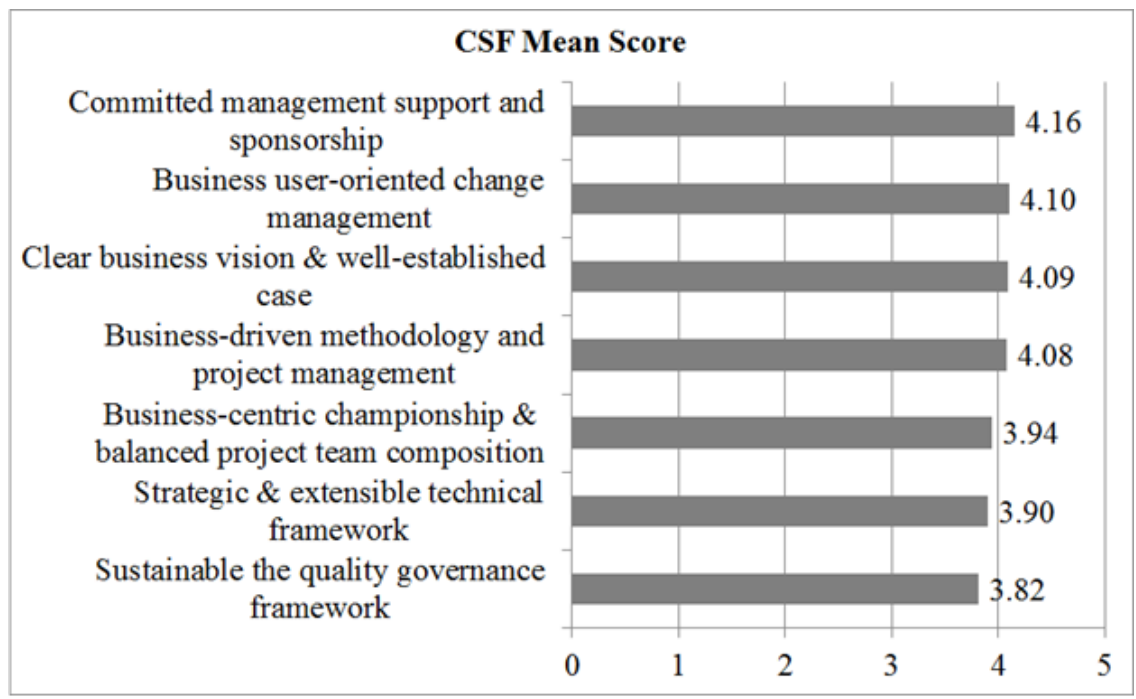

Figure 4. CSF Mean Score

When reflecting on their findings, Yeoh et al. [20] commented, "Notably, data and technical-related factors did not appear to be the most critical ... technological difficulties can be solved by technical solutions. However, it was found that achieving management and organizational commitment for a BI initiative posed the greatest challenge..." 


\section{Issues in Information Systems \\ Volume 14, Issue 1, pp.376-384, 2013}

During follow-on case study research in 2010, Yeoh and Koronios [19] found a firm that experienced BI failure did so because, "The business needs and requirements for the BI system had not been clearly defined..." Conversely, firms which were successful in their BI implementations "... Shifted their focus from a technological one and instead adopted an approach to put their respective business needs first." In their conclusion, Yeoh and Koronios [19] stated,

... without a clear business-driven objective, the BI initiatives rarely produce substantial impact on business. As a result, the implementation of a BI system has a much greater likelihood of success when specific business needs are identified at the outset, and when those needs are used to direct the nature and scope of the implementation effort.

Olszak and Ziemba [15] noted the versatility and complexity of modern BI requires the use of a solid methodological foundation and proven scientific theories to improve the likelihood of implementation success. Further, they recommended the theory of critical success factors as a methodology to be followed during implementation [15]. Their research on CSFs for BI system implementation in small and medium size enterprises (SMEs), conducted in 2012, involved a literature review, critical thinking, inductive reasoning, and in-depth interviews with 20 subject matter experts [15]. Olszak and Ziemba [15] included both determinants and barriers to $\mathrm{BI}$ implementation in their findings.

The top three determinants of BI success in SMEs were:

1. The price of the BI system and its implementation (18 respondents).

2. Suitability of the BI for users business needs ( 17 respondents).

3. Integration of BI with operational systems (17 respondents).

Olszak and Ziemba [15] noted, "The biggest barriers that the respondents encountered during the implementation of BI systems have business and organizational character." The top three barriers to BI success are listed below:

1. The lack of a well-defined business problem (18 respondents).

2. The lack of manager support (17 respondents).

3. The lack of knowledge about BI systems and their capabilities (16 respondents).

In summary, for a BI project to be successful, Olszak and Ziemba [15] claimed, "[The] BI system must be part of the company's business strategy. It must correspond to the real needs of users and support key processes in business decisions at all levels of management (strategic, tactical and operational)."

Sen et al. [18] sought to develop a Data Warehouse Process Maturity (DWP-M) model that guided both the implementation and operation of a data warehouse. Version 1.0 of their five-level DWP-M model, patterned after the software capability maturity model (CMM), was published in May 2004 following a workshop with industry experts [18]. Figure 5 is this researchers graphic rendering of the version 1.0 textual DWP-M model. The DWP-M model included a tentative list of 29 Key Process Areas (KPAs) on which the organization should focus to improvement efforts [18]. 


\section{Issues in Information Systems}

Volume 14, Issue 1, pp.376-384, 2013

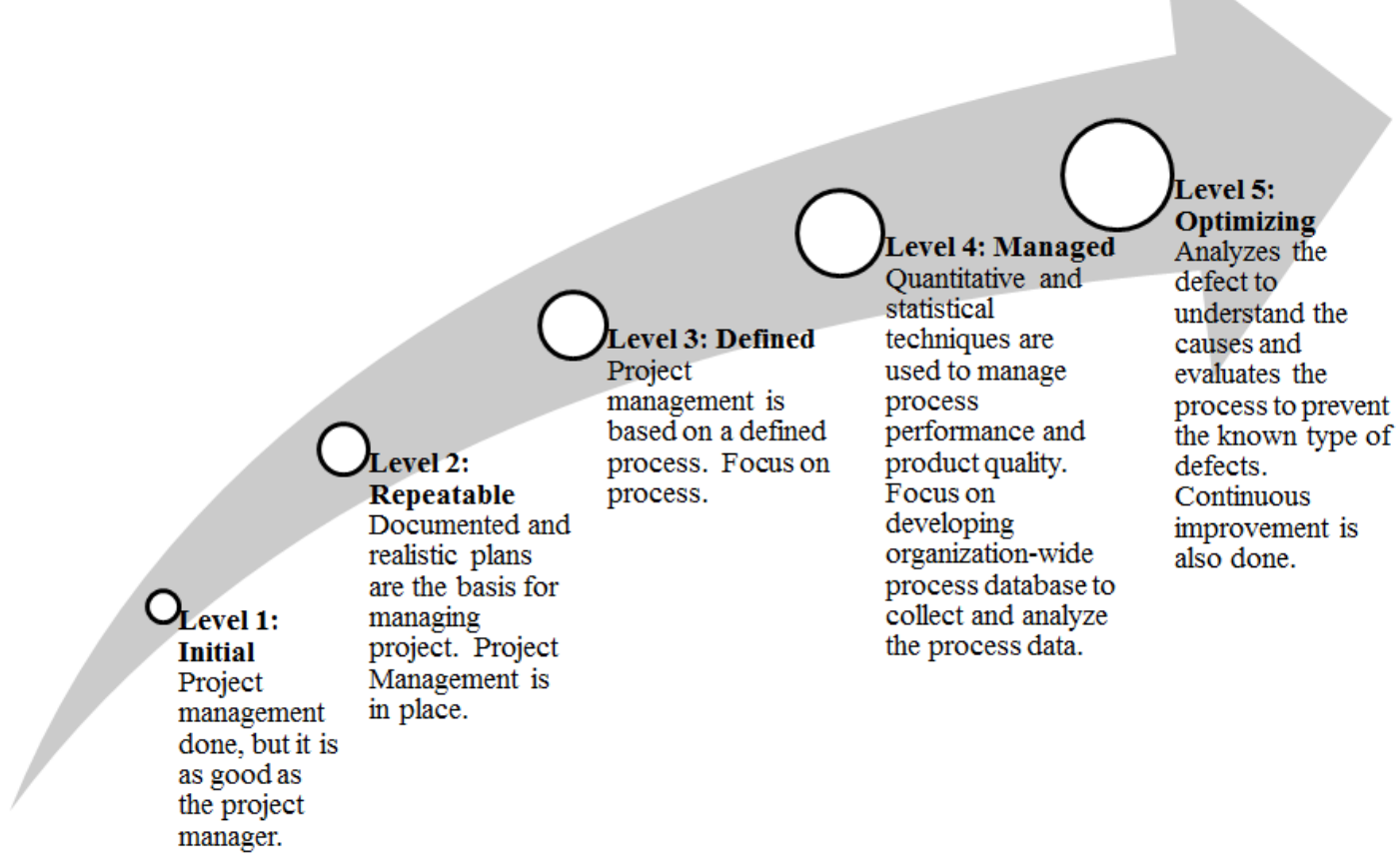

Figure 5. Data Warehouse Process Maturity Model

In March 2006, after nine revisions, version 3.0 of the DWP-M model was presented to a group of experts [18]. Sen et al., [18] commented, "At the end of this session, the group appeared to have reached closure. While assigning the KPAs to different maturity levels, the workshop participants also identified the dependencies among them." Figure 6 is an adaptation of their maturity level two KPA relationship map.

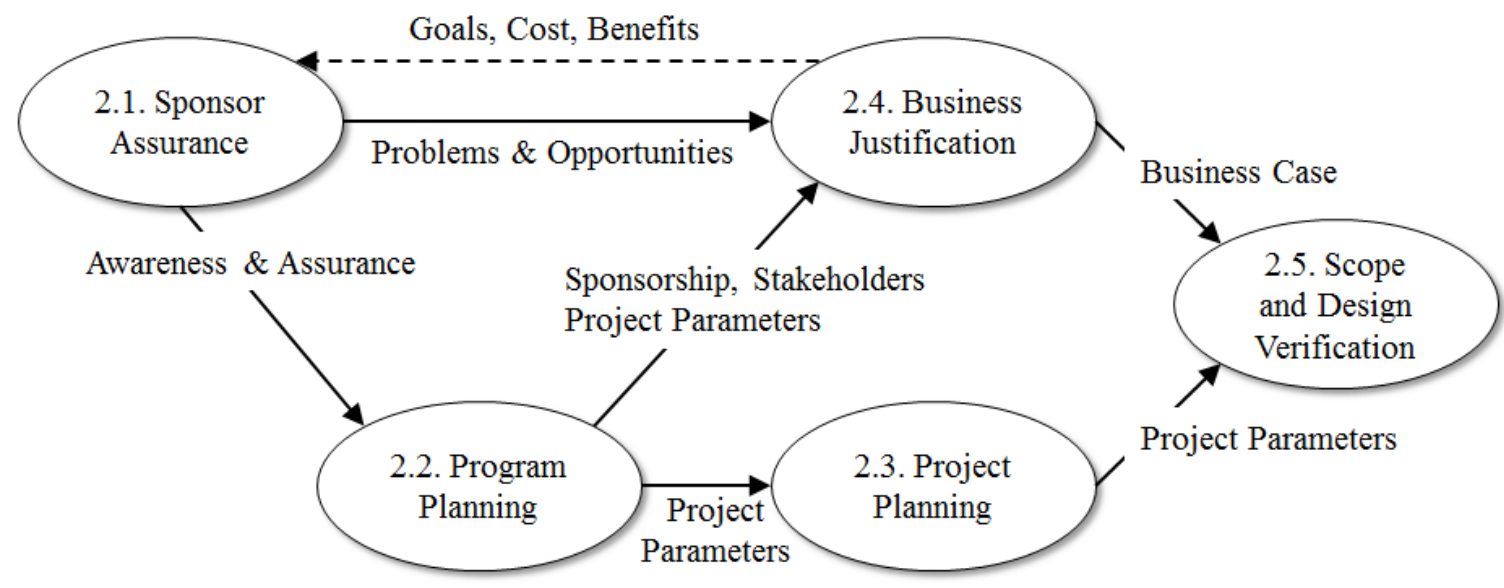

Figure 6. Level 2 Key Process Area Relationship Diagram

The direction of the arrows was significant. An arrow pointing from one activity to another indicated the first activity was a predecessor, or antecedent, to the second. Reflection on Figure 4 yielded two observations. First, the overall flow from antecedent to outcome was from left to right. Second, key process area 2.1 Sponsor Assurance was foundational to the four remaining key process areas. The arrows leaving key process area 2.1 underscored the importance not only of sponsor's engagement (in the form of awareness and assurance) with the data warehouse 


\section{Issues in Information Systems \\ Volume 14, Issue 1, pp.376-384, 2013}

initiative but also of the sponsor's ability to articulate the (business) problems and opportunities the data warehouse must address.

Sangar and Iahad [17] also emphasized the need for a well-defined business problem when they commented, "... the intelligence needs of decision-makers must be defined by discovering the key intelligence topics and questions ..." Their research, published in 2013, developed a conceptual framework to guide BI system implementations [17]. There CSFs were divided into two groups, managerial and technical, and were organized by a three-stage (preimplementation, implementation, post-implementation) implementation model [17].

\section{Convergence On The Root Cause Of Failure}

The causes of data warehouse implementation challenges appear to converge on one primary root cause; namely not understanding the user's business problem. MacDonald [13] observed, "Identifying a critical business problem is not always easy. The business problem selected must have senior management backing which correlates to a willingness to solve the problem." Connor [4] shared a similar view when he stated, “...customers don't meet endusers expectations, they don't have a clear view of the business problem they want to use the data warehouse for ..." Gartner Research [8] echoed this primary root cause in the following quote:

"It is hard to believe that IT organizations still build data warehouses with little or no business involvement," said Frank Buytendijk, research vice president at Gartner. "But some IT experts still believe it is important to 'anticipate the needs of the users.' They also suffer from the 'Atlas Syndrome' - trying to carry the weight of the world on its shoulders - solving problems the users 'do not understand.' As valid as this may seem, it results in a negative outcome."

The CSF with the highest mean score of Hwang and Xu [10] was "clearly defined business needs/benefits." Likewise, Yeoh and Koronios [19] found a cause for BI failure was, "the business needs and requirements for the BI system had not been clearly defined ..." Similarly, Olszak and Ziemba [15] found the top barrier to successful BI implementation was, "the lack of a well-defined business problem." The data warehouse process maturity model level two KPA relationship map of Sen et al. [18] identified sponsor assurance, which included the sponsor's ability to articulate the business problem an opportunity, as foundational to the four remaining processes. Finally, Sangar and Iahad [17] claimed understanding the intelligence needs of decision-makers was the first step in developing a BI process.

In summary, for a data warehouse to succeed, it must provide data, information, and knowledge that allow a user to solve their business problems. More important than building the system right (i.e. CSFs for data warehousing implementation), is building the right system (i.e. providing a solution to the business problem). To solve a user's business problem, the IT analyst must understand the problem. To understand the business problem, the analyst must engage the user, elicit their problem, and agree together on the solution.

\section{Rockart's Classic CSF Method}

Rockart [16] also observed the problem of defining concisely exactly what information senior manager's required. In response he created the CSF method to make needs explicit. The implementation of Rockart's CSF method involved two, and possibly three, interviews with the chief executive [16]. The objective of the first interview was: to understand the executive's goals, to define the CSFs supporting the goals, to identify an initial set of measures for the CSFs, and to consider combining, restating, or eliminating CSFs [16]. During the second interview, results of the first interview were reviewed; the CSFs were "sharpened up;" and measures and reports showing data/information were discussed in detail [16]. The third, and final interview, was only required if the first two failed to obtain concurrence on the CSFs, measures-and-reporting sequence [16]. Figure 7, developed by this researcher, is a graphical representation of Rockart's CSF method. 


\section{Issues in Information Systems}

Volume 14, Issue 1, pp.376-384, 2013

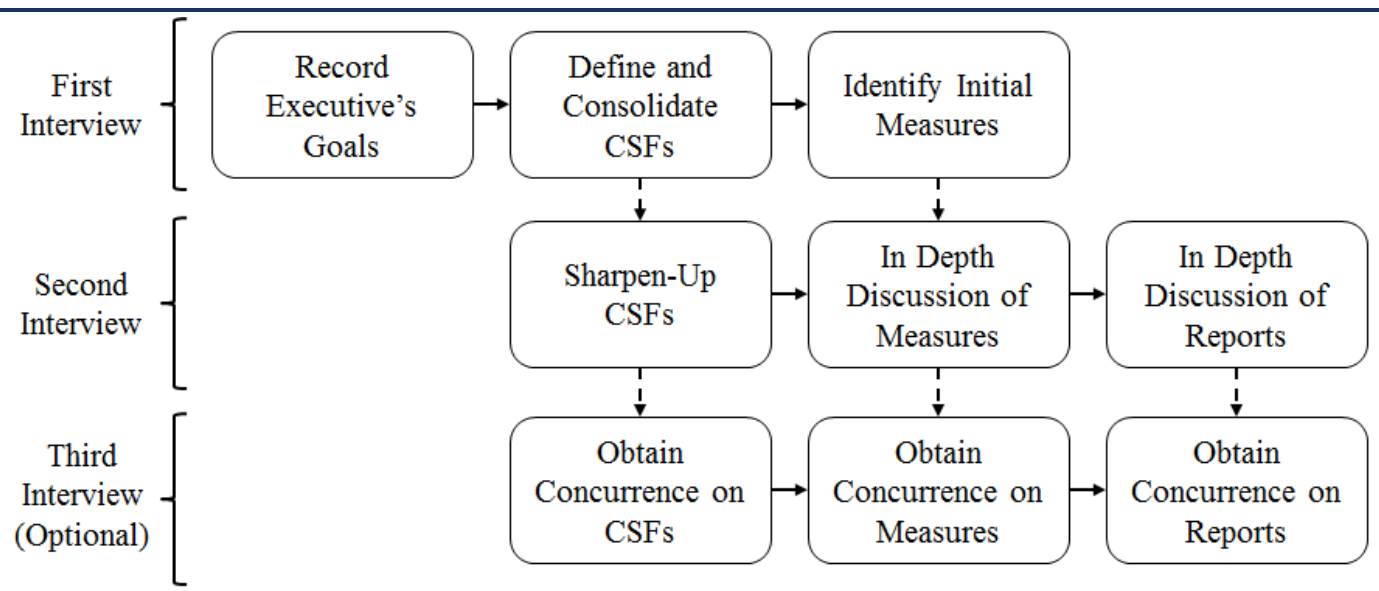

Figure 7. Rockart's CSF Method

While the focus of Rockart's article was on the chief executive, he did note that CSFs would be useful at each level of general management [16]. Boynton and Zmud [1] recommend the population include a diagonal slice of the organization; that is eliciting CSFs from not only different management levels, but also different functional disciplines, thus reducing the potential for bias. Rockart [16] identified the following benefits likely to accrue by cascading CSFs through the layers of management:

- Helping the manager to determine factors on which to focus their attention.

- Forcing managers to develop good measures for CSFs.

- Clearly defining information needs and avoiding costly collection of more data than is required.

- Moving away from reporting data that is "easy to collect" toward collection of data significant for the success of the particular management level.

- Acknowledging that some factors are temporal and, consequently, information systems must change to meet business needs.

- The usefulness of CSFs extends beyond information system design to information system planning. Further, CSFs can be arrayed hierarchically and used as an important vehicle of communication for management.

In 1981 Bullen and Rockart [2] published a working paper titled "A Primer on Critical Success Factors" which allowed researchers to gain a deeper understanding of Rockart's original CSF method. Organized in four sections, the primer provided: an introduction; definitions and concepts; an interview procedure; and recommendations for the analysis of data. Looking back over the history of CSFs, Cooper [5] observed,

Since its initial inception, the CSF method has been adapted and extended to meet the needs of a wide range of research projects including: extensions to the domain and industries of applications; adaptations to the techniques used to elicit CSFs; and extensions to ways in which the results of CSF studies are presented.

In summary, Rockart's CSF method, and the enhancements to this method made by many researchers, can be productively applied to the fundamental challenge of data warehousing; understanding the user's business problems and providing concise, dependable, data, information, and knowledge, about current operations, trends, and changes.

\section{CONCLUSIONS}

This article began by describing the pressures placed on business by an increasingly complex business environment. In response to this pressure, businesses need the right data to make strategic, tactical, and operational decisions. This data, however, is often stored in fragmented functional systems and must be accessed, integrated, an organized into a form that is consistent, reliable, timely, and readily available, wherever, and whenever needed. In short, 


\section{Issues in Information Systems \\ Volume 14, Issue 1, pp.376-384, 2013}

fragmented data must be moved to a data warehouse where it is transformed into information (organized data) and knowledge (patterns, trends, and/or correlations derived from information).

Data warehouses hold great potential for strategic benefit when implemented well. Unfortunately, up to $50 \%$ of data warehouse implementations end in failure. Several researchers searched for root causes. Their research converged on the primary root cause of not understanding the user's business problems. Clearly and concisely identifying user's problems is not always easy. Fortunately, Rockart provided a Critical Success Factor (CSF) method that has a proven track record of moving from business problems to the data and information needed to support management decisions.

Rockart's CSF method provided a classic response to the relatively modern question; "What methods exist to help assure data warehouse implementation success?"

\section{REFERENCES}

1. Boynton, A. C., \& Zmud, R. W. (1984). An Assessment of Critical Success Factors. Sloan Management Review (pre-1986), 25(4), 17.

2. Bullen, C. V., \& Rockart, J. F. (1981). A primer on critical success factors. Retrieved from http://dspace.mit.edu/handle/1721.1/1988

3. Business intelligence: a managerial approach. (2011) (2nd ed.). Boston: Prentice Hall.

4. Connor, D. (2003). Report: Data warehouse failures commonplace. Network World, 20(3), 24.

5. Cooper, V. A. (2009). A Review of the Critical Success Factor Method Using Insights from an Interpretive Case Study. Journal of Information Technology Case and Application Research, 11(3), 9-42.

6. Daniel, D. R. (1961). Management Information Crisis. Harvard Business Review, 39(5), 111-121.

7. Debons, A. (2008). Information science 101. Lanham MD: Scarecrow Press.

8. Gartner Says More Than 50 Percent of Data Warehouse Projects Will Have Limited Acceptance or Will Be Failures Through 2007. (2005, February 24). Business Wire, p. 1. New York, United States.

9. Heizer, J. H., \& Render, B. (2011). Principles of operations management. Boston: Pearson Education.

10. Hwang, M. I., \& Xu, H. (2005, Fall). A Survey of Data Warehousing Success Issues. Business Intelligence Journal, 10(4), 7-13.

11. Inmon, W. H. (2005). Building the Data Warehouse (4th ed.). Wiley.

12. Kimball, R. (2002). Design constraints and unavoidable realities. Intelligent Enterprise, 5(14), 16-17+.

13. MacDonald, M. (1998). Beat the odds. CMA, 72(5), 16-18.

14. Magal, S., \& Word, J. (2011). Integrated Business Processes with ERP Systems. Hoboken NJ: Wiley.

15. Olszak, C. M., \& Ziemba, E. (2012). Critical success factors for implementing business intelligence systems in small and medium enterprises on the example of upper Silesia, Poland. Interdisciplinary Journal of Information, Knowledge, and Management, 7, 129-150.

16. Rockart, J. F. (1979). Chief executives define their own data needs. Harvard Business Review, 57(2), 81-93.

17. Sangar, A. B., \& Iahad, N. B. A. (2013). Critical Factors That Affect The Success Of Business Intelligence Systems (BIS) Implementation In An Organization. International Journal of Scientific \& Technology Research, 2(2), 176.

18. Sen, A., Ramamurthy, K. (Ram), \& Sinha, A. P. (2012). A Model of Data Warehousing Process Maturity. IEEE Transactions on Software Engineering, 38(2), 336-353. doi:http://dx.doi.org/10.1109/TSE.2011.2

19. Yeoh, W., \& Koronios, A. (2010). Critical Success Factors for Business Intelligence Systems. The Journal of Computer Information Systems, 50(3), 23-32.

20. Yeoh, W., Koronios, A., \& Gao, J. (2008). Managing the Implementation of Business Intelligence Systems: A Critical Success Factors Framework. International Journal of Enterprise Information Systems, 4(3), 79-94. 